Sir,- - I was interested to read the report by Dr. Patricia E. Campbell of a case of sudden death in labour following a penicillin injection (Journal, January 8, p. 87). There are several features about it, however, which make the diagnosis of anaphylaxis seem unsatisfactory.

These are as follows : (1) The time interval of one hour between the injection and collapse of the patient is rather long for intravenous injection of the drug-probably the cause of the majority of sudden deaths after penicillin injection-to have been responsible. (2) Reporting the use of massive doses of penicillin, McLean ${ }^{1}$ records sudden death of three patients, and says, "As death occurred in all cases within twelve hours of the initial dose, it seems unlikely that anaphylaxis was responsible." (3) The case clinically appears to present all the features of amniotic fluid embolism described originally by Steiner and Lushbaugh, ${ }^{2}$ and subsequently by Reid et al., ${ }^{3}$ in the United States of America, and by Shotton and Taylor ${ }^{4}$ in this country, and the necropsy findings are not inconsistent with this condition. Dr. Campbell does not state whether or not the lungs were examined histologically.-I am, etc.,

Glasgow, S.1. GORDON BRILL.

$$
\begin{aligned}
& \text { 1 Practitioner, 1955, 174, } 45 . \\
& 2 \text { J. Amer. med. Ass., 1941, 117, 1245. } \\
& \text { 3. Amer. J. Obstet. Gynec., 1953, 66, 465. } \\
& \text { 4. Obstet. Gynaec. Brit. Emp., 1949, 56, } 46 .
\end{aligned}
$$$$
\text { REFERENCES }
$$

\section{Appendicectomy in Chronic Appendicitis}

SiR,-In common no doubt with the majority of your readers, I have been very interested in the correspondence on appendicectomy for "chronic" appendicitis which followed the publication of the paper by Messrs. I. McLennan and J. K. Watt (Journal, September , 25, 1954, p. 736). I was particularly impressed by the letter from Professor J. Chassar Moir (Journal, December 11, p. 1415) in which he pointed out the fact-shown in their paper-that " chronic" appendicitis appears to be much commoner in females of menstrual age and that, in his opinion, this is usually attributable to disordered ovarian function.

I am in the process of preparing a paper which lends much support to this hypothesis. In an analysis of all cases with pain in the right iliac fossa, as the presenting symptom seen at one particular surgical out-patient clinic at a provincial teaching hospital during the course of 12 months, the following figures were obtained : females 52 ; males 11 ; total 63 . Thirty-seven of the females were between 14 and 30 years of age, the majority being either in the late 'teens or early twenties. These girls were nearly always complaining of intermittent "stabbing" or "niggling" pain in the right iliac fossa (and frequently in the left iliac fossa if specifically asked) which usually lasted anything from a few minutes to several hours, with little or no constitutional upset and usually no abnormal physical signs at the time of examination, although deep tenderness in the right iliac fossa had often been found by their own doctors during or shortly after an attack. In 33 out of the 52 females a diagnosis of ovulation pain was made and the patient reassured. O Of the remaining 19 females, of whom the majority were between 40 and 50 , one had, acute appendicitis and three were thought to be recovering from a recent acute attack, whilst in the remainder a variety of disorders was found, including constipation, renal, gall-bladder, and frank gynaecological disease, plus one pregnancy. Among the 11 males, 6 had a history and/or signs consistent with recent acute or recurrent appendicitis, whilst two were found to be suffering from severe constipation. A right basal lung cyst, a large para-umbilical hernia, and right myofascial lumbar strain completed the list of males. During the same 12 months, 10 females between the ages of 15 and 30 were operated on for "apoplectic" ovary (ruptured lutein cyst) and 8 treated conservatively in the beds of this unit. It is believed that this condition, particularly in a minor form, is by far the commonest cause of recurrent pain in the right iliac fossa in young women.

Appendicular colic, characterized by recurrent central abdominal pain generally lasting only a few minutes at a time and often associated with slight deep tenderness over the appendix but without local rigidity, is a much more plausible explanation for the symptoms relieved by appendicectomy particularly in childhood. In such cases. the appendix is nearly always normal to the eye of the sur- geon and to the unbiased pathologist. It may be kinked and usually contains inspissated faecal matter, but it is rarely chronically inflamed. In so far as constipation appears to be the basic cause of appendicular colic, the condition tends to cure itself in time, but this may extend over many. years. For such cases, appendicectomy is probably a justifiable but certainly not an essential procedure. While chronic appendicitis undoubtedly exists as a pathological entity, and probably as the sequel to acute or recurrent attacks, it is, I think, a comparative rarity, if for no better reason than the vigilance of the average G.P. in referring all suspicious cases of acute or subacute appendicitis to hospital for emergency admission. Clinically, chronic appendicitis is either silent or declares itself only during acute exacerbations. Following the resolution of an appendix abscess, the appendix is histologically chronically inflamed, yet the patient is symptom free unless there is a recurrence of acute inflammation.

My conclusions, for what they are worth, are: (1) Chronic appendicitis is relatively rare and is a sequel to acute inflammation. (2) Chronic appendicitis per se is symptomless. (3) Appendicular colic is common, particularly in children, and is due to inspissation of faecal matter in perhaps a kinked but not usually a chronically inflamed organ. (4) Appendicular colic can be produced only if the wall of the appendix is capable of actively contracting, and this function is inevitably inhibited by fibrosis. (5) Acute-onchronic (or recurrent) appendicitis is characterized more by persistent pain in the right iliac fossa (lasting continuously for several hours or days) and less by initial central colic. (6) Recurrent attacks of pain localized to the right iliac fossa and lasting only short periods (minutes to perhaps an hour) are rarely due to appendicitis. (7) Recurrent niggling pain in the right iliac fossa of young women is generally due to disordered ovarian function.-I am, etc.,

$$
\text { London, w.C.1. A. HenRy Millard. }
$$

\section{Distribution of R.M.B.F. Christmas Gifts}

SIR,- I am sure that your readers will be pleased to learn that the contributions to provide Christmas gifts for the beneficiaries of the Royal Medical Benevolent Fund amounted to $£ 2,775$. We were able to,distribute $£ 8$ to each of them.

The warmth of gratitude expressed in letters of thanks would have gladdened the hearts of the contributors, and of yourself, Sir, for giving space so generously for the publication of the appeal.-I am, etc.,

London, S.W.15.
Royal Medical Benevolent Fund

\section{POINTS FROM LETTERS}

\section{Fixation of Blood Smears}

Mr. N. V. GuRJAR (Bombay) writes: I have been using successfully a $7 \%$ solution of sulphosalicylic acid as a fixative for blood smears. More than 3,000 smears of blood and marrow have been fixed by this method for a couple of seconds and then stained with different haematological stains. The results have been excellent. The cytological morphology is better as compared with alcohol fixation. Another advantage is that smears so fixed can remain (unstained) for any length of time without any fungus growth, which is a common feature of alcohol fixation. Recently I have also fixed some tissues for histological section with $10 \%$ solution of sulphosalicylic acid for 24 hours, and this has shown less shrinkage in cell cytology. This might prove a bit expensive in comparison to formol-saline or Bouin's fixative, but the advantage of getting better morphology could be a consideration particularly when dealing with small biopsies.

\section{The Future of Surgery}

Dr. E. F. BLumberG (London, S.W.3) writes: With reference to Sir Heneage Ogilvie's statement (Journal, December 18, 1954, p. 1435) that " the contributions of Germany to surgical advance in the last 50 years have been pathetic," for the years since 1933 your author is obviously correct. The first 25 years, however produced a vast number of great names. I am including a small collection-Kocher, Trendelenburg, Lexer, Israel, Enderlen, Czerny, Bier (lumbar anaesthesia), Kehrer, Koerte, Garré, Sauerbruch. 